\title{
A PRIORI TRASCENDENTAL Y A PRIORI CONVENCIONAL. EL INTRINCADO CAMINO DE LO $A$ PRIORI DE KANT A PUTNAM
}

\author{
TRANSCENDENTAL A PRIORI AND CONVENTIONAL A PRIORI. \\ THE DEVIOUS PATH OF APRIORITY FROM KANT TO PUTNAM
}

\author{
Pablo Melogno
}

\begin{abstract}
RESUMEN
En diversos trabajos elaborados en los 60's y 70's, Putnam introdujo la noción de a priori contextual, buscando dar cuenta de los principios que marcan los límites de la experiencia posible al interior de un esquema conceptual. Para Putnam, dichos principios se originan en la satisfacción de necesidades cognitivas específicas y no a estipulaciones convencionales. Este trabajo examina la noción putnamiana de lo a priori en relación a las propuestas de Poincaré, Reichenbach y Carnap, buscando establecer en cada caso los puntos de convergencia con la concepción putnamiana, así como los motivos que llevaron a Putnam a rechazar las lecturas convencionalistas del a priori. Se defiende que la propuesta putnamiana constituye una rehabilitación del componente trascendental del a priori kantiano, en cuanto para Putnam los principios a priori no solo son constitutivos de los objetos, sino que fijan límites cognitivos que solo pueden rebasarse en contextos de cambio conceptual.
\end{abstract}

PALABRAS CLAVE: Putnam, Reichenbach, Kant, Carnap, Poincaré, a priori.

\begin{abstract}
In several works produced in the 60's and 70's, Putnam introduced the notion of contextually a priori, trying to give an account of the principles that fix the limits of the possible experience within a conceptual scheme. According to Putnam, these principles are originated by the satisfaction of specific cognitive needs, and not by conventional stipulations. This paper discusses the putnamian notion of the a priori in relation to the stances of Poincaré, Reichenbach and Carnap, trying to establish in each case the points of convergence with the putnamian conception, as well as the reasons that led Putnam to reject the conventionalist views of the a priori. It is argued that the putnamian account can be considered as a rehabilitation of the transcendental component of the Kantian a priori, as for Putnam the a priori principles
\end{abstract}


are not only constitutive of the objects, but they fix cognitive limits that can only be overcome in contexts of conceptual change.

KEYWORDS: Putnam, Reichenbach, Kant, Carnap, Poincaré, a priori.

\section{INTRODUCCIÓN}

Los problemas del conocimiento a priori constituyen uno de los principales focos de interés de la filosofía de Putnam en las décadas de los 60's y 70's. En varios escritos Putnam intentó dar cuenta del status y el alcance de las proposiciones a priori, así como de las condiciones que permiten tanto su adopción como su revisión. La consideración putnamiana de los problemas de la aprioridad se nutre de dos vertientes. Por un lado, la polémica con Quine en torno a la distinción analítico-sintético; por otro, el problema de la función de los principios a priori en las teorías científicas, que había sido inaugurado por Kant y continuado entre otros por von Helmholtz, Poincaré, Cassirer, Natorp, Schlick, Reichenbach y Carnap.

En este trabajo emprendemos una revisión de la segunda vertiente, buscando clarificar la noción putnamiana de lo a priori en relación a las propuestas que la anteceden. Para ello examinaremos la imagen del conocimiento a priori en Poincaré, Reichenbach y Carnap, buscando establecer en cada caso los puntos de convergencia y discrepancia con la propuesta putnamiana. Nos centraremos en el período que va desde la publicación de "Lo analítico y lo sintético" de 1962, hasta, "Analyticity and Apriority: Beyond Wittgenstein and Quine" de 19791.

Si bien el período seleccionado no arroja una imagen homogénea de lo a priori, muestra sí una agenda de problemas e inquietudes filosóficas lo suficientemente definida para constituir una unidad de análisis. Esto no sucede con el pensamiento posterior de Putnam, ya que a partir de Raqón, verdad e historia (1981) tanto la agenda filosófica de Putnam como sus tesis específicas sufren fuertes modificaciones, lo que requeriría para este período una consideración por separado. Además se trata del período de mayor interés por parte de Putnam en los problemas de la aprioridad, ya que durante la década de los 80's su pensamiento se orientará más bien a los problemas del realismo. Finalmente, entendemos que si bien la literatura secundaria sobre Putnam es por demás copiosa, aún queda algo por decir sobre su conexión con el neokantismo, el empirismo lógico y el mismo Kant, y la con-

1 Excepto por menciones puntuales a escritos posteriores (Putnam 1981, 1994, 2015). 
cepción putnamiana de lo a priori constituye una muy prometedora vía para llevar esto a cabo.

La indagación de las proposiciones a priori que emprende Putnam sigue la estela de Poincaré y Reichenbach, en lo que hace a la variabilidad de los principios a priori y las condiciones que habilitan su revisión. Pero mantiene una impronta neokantiana que la distancia de lecturas convencionalistas como las de Poincaré o Carnap, en cuanto enfatiza el papel de los principios a priori como condiciones de posibilidad del conocimiento. Sobre esta base trataremos de mostrar que la noción putnamiana de lo a priori opera una rehabilitación parcial pero significativa de la filosofía de Kant, en cuanto niega el carácter convencional de los principios a priori y reivindica su función como límite cognitivo de los sujetos. Para ello realizaremos un recorrido por las caracterizaciones del conocimiento a priori que más peso tienen en los desarrollos de Putnam, comenzando por el convencionalismo de Poincaré.

\section{EL A PRIORI CONVENCIONAL DE POINCARÉ}

En la Crítica de la Razón Pura - KrV - Kant consideró a los juicios a priori como constitutivos del conocimiento, necesarios (A XV) y anteriores a la experiencia (B XVI-XVII). El ser constitutivo del conocimiento no es separable del ser necesario, ya que el carácter constitutivo de los juicios a priori implica que son condición de todo conocimiento posible para todo sujeto y en todo tiempo. Esto cancela la posibilidad de que haya distintos principios que puedan valer como $a$ priori en contextos diferentes. En términos de Kant, los principios a priori son trascendentales, en cuanto fijan las condiciones de posibilidad del conocimiento, al menos en los seres humanos.

Kant entendió a su vez que la geometría de Euclides y la física de Newton eran la expresión consumada de las capacidades de la razón (B X), por lo que los principios de la geometría euclidiana no solo cuentan como descripciones acerca de cuerpos y figuras geométricas, sino como expresión invariante de la forma en que los seres humanos nos representamos el espacio (B 38-39). Los axiomas de Euclides son impuestos como resultado de nuestras capacidades de conocimiento, y esto los convierte en un caso privilegiado de conocimiento a priori.

Esta noción kantiana de lo a priori sufre una fuerte revisión a partir del surgimiento de las geometrías no euclidianas — Gne_- proceso que dará lugar a un cambio en la consideración de los principios geométricos como verdades necesarias. El impacto filosófico de las Gne llevará a poner en cuestión el carácter autoevidente de los principios de la geometría, lo que terminará afectando a la idea misma del conocimiento a priori como necesario e invariante. 
Existe cierto consenso ${ }^{2}$ en que el trabajo de Hermann von Helmholtz constituye uno de los primeros intentos por evaluar el impacto de las Gne en la filosofía de Kant, dando lugar a miradas críticas sobre el a priori trascendental kantiano, que encontrarán su expresión más influyente en el convencionalismo de Poincaré3. Este constituye una tentativa de preservar la idea kantiana de que los principios de la geometría son a priori, pero defendiendo al mismo tiempo su carácter contingente y la posibilidad de elección entre principios alternativos.

La variabilidad del conocimiento a priori aparece en Poincaré considerada mayormente en la geometría y la mecánica, ya que mantiene que los axiomas de la aritmética sí son juicios sintéticos a priori, (1905, xx, 57) ${ }^{4}$, y por lo tanto son necesarios. Pero en relación a los axiomas geométricos, Poincaré entendía que no son proposiciones empíricas, leyes lógicas, ni juicios sintéticos a priori. Si se acepta a Kant que es imposible concebir la falsedad de un juicio sintético a priori, no podríamos concebir la falsedad de los axiomas de Euclides, ni jamás habríamos desarrollado geometrías alternativas. Pero una vez que existen las Gne los axiomas geométricos no pueden tomarse como sintéticos a priori, ya que si lo fueran "Nos serían impuestos con una fuerza tal que no podríamos concebir la proposición contraria, ni construir sobre ella un edificio teórico. No habría geometría no-euclidiana." $(1905,57)^{5}$.

Los axiomas geométricos deben considerarse más bien definiciones disfrazadas, en cuanto su contenido es resultado de una estipulación del significado de los términos básicos de cada sistema geométrico. Esto implica que los términos básicos no tienen un significado pre-teórico anterior al sistema axiomático al que pertenecen, por lo que no hay una forma independiente — ej. la intuición- de comprender su contenido ni de establecer su verdad. De aquí que para Poincaré los axiomas no cuentan como proposiciones en sentido estricto, sino que son resultado de decisiones metodológicas respecto a cómo utilizar determinadas palabras. Esto impide considerar que haya una serie de axiomas intrínsecamente necesarios,

2 Coffa (1991), Paty (1992), Friedman (2002), DiSalle (2006), Carus (2007), Mormann (2007). 3 Señala Peláez que tanto Helmholtz como Poincaré "se vieron en la tarea de congeniar la idea básica kantiana de que el espacio ha de ser una forma subjetiva a priori que condiciona la experiencia perceptiva espacial, con el rechazo a cualquier determinación a priori de dicha estructura." (2008b, 103).

${ }^{4}$ Folina (2014) ha señalado que Poincaré otorga a las convenciones de la geometría un a ubicación muy específica, intermedia entre los juicios sintéticos a priori de la matemática pura, y los juicios a posteriori de las ciencias físicas.

${ }^{5}$ La traducción es nuestra en todos los casos en que la referencia aparece en inglés. 
y permite poner en pie de igualdad distintas geometrías posibles. Implica también que la verdad en geometría sólo se predica de las proposiciones obtenidas dentro de un sistema, ya que no de los principios que permiten construir el sistema.

Como veremos más adelante, Putnam asimila las ideas de Poincaré a través de Carnap y Reichenbach, y al igual que estos, hereda la posición de que los principios a priori son variables y a la vez constitutivos del conocimiento. Pero a diferencia de Poincaré niega que sean convencionales, considerando que los principios que cuentan como a priori dentro de un marco lingüístico mantienen dicho status como resultado de las constricciones que operan sobre nuestros procesos de conocimiento, y no como producto de elecciones entre alternativas igualmente legítimas.

Claro está que para Poincaré el carácter convencional no hace que la elección de los principios de la geometría sea arbitraria o irrestricta, sino que es más bien 'guiada por la naturaleza' y por el requisito de coherencia: "Nuestra elección entre todas las convenciones posibles es guiada por los hechos experimentales; pero permanece libre, y sólo está limitada por la necesidad de evitar toda contradicción..." (Poincaré 1905, 58-59). Las convenciones 'permanecen libres' en cuanto ni la lógica, ni la experiencia, ni la intuición nos conducen a adoptar necesariamente un determinado conjunto de convenciones, aunque tampoco nos permiten adoptar cualquier convención ${ }^{6}$.

Cabe aquí adelantar otra diferencia de relieve entre Putnam y Poincaré. Para Putnam los componentes a priori del conocimiento no son resultado de ninguna elección, lo que cancela el escenario convencionalista en el cual nos enfrentamos a distintos conjuntos de principios, todos igualmente elegibles. En un marco putnamiano no es posible afirmar que "Lo que protege a tales principios de la revisión no es su certeza apodíctica, sino nuestra decisión de tratarlos como irrevisables." (DiSalle 2006, 86), sino que más bien debemos afirmar que lo que protege a ciertos principios no es su certeza apodíctica sino nuestra imposibilidad de concebirlos como irrevisables. Para Putnam determinados principios cuentan como a priori en un cier-

${ }^{6}$ DiSalle (2006) cuestionó que el carácter a priori que Poincaré le otorga a los axiomas geométricos impide dar cuenta de su permeabilidad a la experiencia. Folina (2014) trató de solucionar el punto señalando que para Poincaré los principios de la geometría no son completamente a priori, lo que permite que respondan a la experiencia. Veremos que en Putnam el panorama es más claro, en cuanto los principios a priori no son abandonados como consecuencia de resultados empíricos, sino como consecuencia del desarrollo de una teoría articulada sobre principios alternativos. 
to contexto dado porque no podemos concebir su falsedad, y en ese sentido no elegimos otorgarles valor a priori, sino que este valor — podemos decir kantianamente- se nos impone.

La discusión del a priori convencional de Poincaré será una referencia obligada en el horizonte neokantiano de los primeros empiristas lógicos. Los abordajes de Reichenbach y Carnap constituyen las principales referencias que tomaremos para dar cuenta de esta nueva concepción del a priori, que ejercerá una duradera influencia en el pensamiento de Putnam.

\section{EL A PRIORI RELATIVIZADO DE REICHENBACH}

Friedman (1999), ha destacado que los empiristas lógicos rechazaron la visión de Kant por entenderla incompatible con el desarrollo de la geometría y la física de comienzos del siglo XX, pero manteniendo la idea de que existen ciertos principios no-empíricos y a la vez revisables ${ }^{7}$. Señala también que el problema principal que la teoría general de la relatividad planteó a pensadores como Reichenbach, Schlick y Carnap, consistía en cómo coordinar un sistema matemático altamente formalizado como el relativista con la realidad física (Friedman 2002, 2007). En este marco el trabajo de Reichenbach representa una novedosa reformulación de lo a priori, y un intento ambicioso de evaluar las consecuencias filosóficas de la relatividad ${ }^{8}$.

Reichenbach entiende que el desarrollo de la relatividad impide aplicar la geometría euclidiana a la física, poniendo en tela de juicio el carácter necesario de los axiomas de Euclides. Una vez que la concepción kantiana del espacio como intuición pura a priori presupone la validez de los axiomas de Euclides, el desarrollo de la relatividad constituye una refutación de la teoría kantiana (1920, 4-5). Reichenbach pretende establecer qué aspectos del a priori kantiano deben ser abandonados como consecuencia de la relatividad y cuáles pueden preservarse en un marco relativista. Para esto señala que en Kant la expresión 'a prior' tiene dos

\footnotetext{
7 Peláez (2008b) ha caracterizado este proceso como una 'liberalización del a prior', que dio como resultado una caracterización de los principios a priori como constituyentes puramente formales del conocimiento, y por tanto carentes de contenido fijo.

8 Nos centraremos aquí completamente en Theory of Relativity and a Priori Knowledge, no solo por ser el primer intento sistemático de Reichenbach por discutir estas cuestiones, sino por resultar el más adecuado para mostrar su influencia en el pensamiento de Putnam. En los trabajos de Coffa (1991), Friedman (1999), Ryckman (2003) y Peláez (2008b), se explora con detalle el contexto previo del pensamiento de Reichenbach, así como su posterior orientación al convencionalismo.
} 
sentidos. Por un lado, la aprioridad se vincula con el carácter necesario e invariante de determinados principios, y por otro lado da cuenta de su carácter constitutivo del conocimiento; pero se trata de niveles diferentes. Los principios a priori son constitutivos en cuanto determinan los objetos de las ciencias, pero esto no implica que sean necesarios, sino que pueden modificarse con los resultados de la investigación científica, "Mientras que la teoría de la relatividad arrojó la conclusión de que los principios constitutivos deben ser cambiados, Kant creyó que en tal caso llegaría a su fin todo el conocimiento." (Reichenbach 1920, 71). Reichenbach independiza así el carácter constitutivo de la necesidad, en cuanto 'a prior' ya no significa 'necesario' ni 'invariante', sino solamente 'constitutivo del conocimiento'.

El núcleo de esta nueva versión de lo a priori es el concepto de 'coordinación', que Reichenbach utiliza para explicar cómo se enlazan los objetos físicos con las ecuaciones matemáticas. La coordinación es una condición del juicio cognitivo, de modo similar a la sintesis en Kant'. Para Reichenbach no hay elementos empíricos básicos en el proceso de conocimiento; los objetos del conocimiento físico no están dados previo a las ecuaciones matemáticas, sino que se individualizan como resultado de su introducción. La coordinación es el proceso por el que estas herramientas matemáticas predefinidas se conjugan con la clase indefinida de las percepciones empíricas, y así conforman los objetos de conocimiento: "Entonces ocurre que los objetos individuales y su orden es definido por las leyes físicas. La coordinación en sí misma crea una secuencia de elementos a ser coordinados." (Reichenbach 1920, 42)

Para Peláez (2008b) este proceso de constitución marca una afinidad entre la propuesta de Reichenbach y el realismo interno de Putnam (1981), ya que en ambos casos hay herramientas conceptuales (constitutivas y también variables) operando sobre la materia prima de la experiencia. Ahora bien, que todo objeto de conocimiento cuente como tal sólo dentro de un marco que permite constituirlo, no implica —ni en Reichenbach ni en Putnam- que los objetos sean estériles para poner en cuestión el sistema de conocimiento que permitió constituirlos. Dicho de otro modo, el que la experiencia esté determinada por nuestras categorías conceptuales no hace que dichas categorías resulten inmunes a la experiencia. Más

\footnotetext{
9 “Del mismo modo que el 'análisis de la razón’ en la Estética trascendental de Kant está enfocado en demostrar que el conocimiento resulta solo de una síntesis entre diferentes fuentes de cognición, el 'método de análisis de la ciencia' de Reichenbach muestra cómo esta síntesis debe ser ahora analizada en términos de sus constituyentes 'objetivos' y 'subjetivos.'. (Ryckman 2003, 176)
} 
adelante indagaremos en las razones que permiten a Reichenbach defender esto, y en la sección 5 revisaremos las de Putnam.

Considera Reichenbach que los axiomas de coordinación ofician como principios básicos del conocimiento, en cuanto definen las clases de objetos a los que se aplican las ecuaciones. Se distinguen de los axiomas de conexión; término acuñado para referirse a las leyes físicas en el sentido tradicional, basadas en la detección de regularidades empíricas (1920, 52-55). Mientras que los axiomas de conexión establecen relaciones entre variables definidas, como por ejemplo entre la energía cinética y la temperatura de un gas, los axiomas de coordinación fijan las reglas para establecer relaciones entre objetos, ya se trate de relaciones de proporcionalidad, correlación estadística u otro tipo. Los axiomas de Euclides por ej., cumplen una función de coordinación en la física de Newton, mientras que las tres leyes del movimiento cumplen una función de conexión.

El caso de la adopción del espacio de Minkowsky en la relatividad especial y de la variedad de Riemann en la relatividad general, constituyen episodios en los que el desarrollo de la investigación científica obligó a modificar los componentes constitutivos de las teorías. Más aún, el desarrollo de la relatividad no solo implica un cambio en los axiomas de coordinación, sino también en el status de las reglas métricas que conforman la base de la física, "Estas reglas métricas se vuelven dependientes de la totalidad del mundo de cuerpos circundante. Lo que inicialmente fue llamado un método matemático de razonamiento se ha transformado en una propiedad especial del objeto y de su incrustación en la totalidad de los cuerpos. La métrica ya no es un axioma de coordinación, sino que se ha transformado en un axioma de conexión." $(1920,100)^{10}$.

En la relatividad general el espacio se define mediante una variedad de Riemann que no admite tres opciones de curvatura constante - negativa, positiva, o neutra-, sino una sola opción de curvatura variable, cuya variación depende —a nivel empírico- de la distribución de la materia en el espacio. Pero si la curvatura deja de ser una presuposición geométrica previa a la física, y pasa a ser el resultado de la distribución espacial de la materia, entonces la cuestión de qué geometría se adapta mejor al espacio físico ya no se ubica en el sistema de coordinación, sino en los axiomas de conexión.

El cambio operado por la relatividad en la base geométrica de la física deja la enseñanza de que si bien los axiomas de coordinación no están regularmente

10 Un análisis pormenorizado de cómo Reichenbach pretende dar cuenta del cambio métrico operado en la relatividad se encuentra en Ryckman (1996). 
expuestos a contrastación empírica, sí quedan expuestos a revisión bajo ciertas condiciones específicas de cambio teórico. Entendía Reichenbach que si consideramos los axiomas de coordinación por separado, cada uno de por sí no tiene consecuencias empíricas, pero si tomamos como referencia grupos de axiomas que en un determinado contexto conforman el sistema de coordinación de una teoría, el grupo sí tiene implicancias empíricas, que se ponen de manifiesto cuando ocurre un cambo de teoría que modifica la base de coordinación. Así llega a la novedosa idea de que es la experiencia quien decide lo que cuenta como necesario en un sistema de conocimiento: "No es por tanto posible, como Kant creía, singularizar en el concepto de objeto un componente que la razón considera como necesario. Es la experiencia la que decide qué elementos son necesarios." (Reichenbach 1920, 88)

Reichenbach considera que toda teoría física está asociada a un grupo de transformaciones que delimita los sistemas de coordinación admisibles. En la física de Newton, el espacio-tiempo newtoniano cumple la función constitutiva a priori, y el grupo de Galileo define las transformaciones; en la relatividad el espacio tiempo de Minkowsky es constitutivo, y el grupo de Lorentz provee las transformaciones. Una vez que en cada teoría hay un grupo invariante y un conjunto de sistemas coordinados igualmente admisibles, la elección de un sistema tiene un componente arbitrario, aunque esto no resulta suficiente para atribuir a Reichenbach una postura convencionalista.

No se trata de convenciones en cuanto — contra Poincaré - entiende Reichenbach que si bien cada axioma tomado en asilamiento resulta arbitrario e inmune a la experiencia, puestos en conjunto los axiomas sí quedan expuestos a refutación empírica, y en esa medida no valen como convencionales. A su vez, si la experiencia puede tirarlos abajo entonces se trata de juicios sintéticos por su relación con el contenido empírico, y a priori por su carácter constitutivo. Por último, si los axiomas fueran convencionales sería posible estipular por convención la geometría de Euclides como base de la relatividad general, y en un sentido más general sería posible ensamblar cualquier geometría con un sistema físico dado. Pero el desarrollo de la relatividad muestra que esto no es posible, por lo que la adopción de un conjunto de axiomas no puede considerarse como resultado de una convención $(1920,47 \text { y ss. })^{11}$. De esta manera, los axiomas de coordina-

11 Schlick (1922) había terminado en un auténtico atolladero con este problema, defendiendo que el carácter convencional de la geometría autoriza a mantener la geometría de Euclides como base de la relatividad general, pero dadas las enormes complicaciones que ello traería a la teoría física, resulta más conveniente optar por una base geométrica no euclidiana. 
ción cuentan como juicios sintéticos a priori en sentido kantiano, excepto porque no son necesarios. Reichenbach sigue aquí la idea kantiana de que las categorías 'crean' el objeto, y por esto es posible afirmar que los principios de coordinación son a priori en cuanto constitutivos de la experiencia.

En trabajos posteriores Reichenbach abandona su versión original de lo a priori para orientarse a tesis más cercanas al convencionalismo de Poincaré. Pero al menos en 1920 el a priori queda desligado del status convencional y de la posibilidad de que los sujetos elijan entre diferentes marcos, porque los principios a priori imponen condiciones de conocimiento que solo pueden revisarse en contextos de cambio teórico. Reichenbach mantiene la dimensión del a priori kantiano en cuanto constitutivo del conocimiento, y — contra el convencionalismo- considera que los principios a priori no son producto de una elección. En las secciones 5 y 6 veremos cómo la idea de que los principios a priori pueden ser constitutivos, sumada a la intuición de que sólo varían bajo circunstancias muy singulares, será una poderosa influencia para la noción de a priori contextual de Putnam. Pero pocos años después de la publicación del trabajo de Reichenbach, el tema recibirá otro vigoroso tratamiento en manos de Carnap, que revisaremos a continuación.

\section{CARNAP: APRIORIDAD Y REGLAS DE CONSTITUCIÓN EN AUFBAU}

Peláez (2008b) ha identificado dos concepciones de lo a priori en la filosofía de Carnap. La primera basada en la idea de constitución de la experiencia, apegada al neokantismo y a Poincaré, aparece en la tesis doctoral Der Raum de 1922, y en La construcción lógica del mundo de 1928, popularizado como Aufbau por el título en alemán (tb. Friedman 2007). La segunda conlleva el desplazamiento de Carnap hacia una concepción netamente semántica de lo a priori, y toma forma en La Sintaxis Lógica del Lenguaje de 1934. Aquí nos centraremos en el texto de 1928, no solo porque permite mostrar con detalle las diferencias entre Carnap y Reichenbach, sino porque buena parte de las conexiones entre Carnap y Putnam pueden encontrarse en Aufbau.

$\mathrm{Al}$ igual que Reichenbach, Carnap consideró los problemas de la aprioridad tomando como referencia directa a Poincaré (Carus 2007), y desarrollando una perspectiva convencionalista originada en los problemas de la geometría (Mormann 2007). Aufbau presenta el conocido 'sistema de constitución' para la reconstrucción racional de los conceptos del lenguaje científico. Por 'constituir', entiende Carnap la derivación de todos los conceptos de la ciencia a partir de una serie inicial de conceptos básicos ( $(1)$. Al diferenciar distintos niveles conceptuales, el sistema 
permite expresar todas las proposiciones contenidas en un nivel de conceptos mediante los conceptos del nivel más básico, "Por sistema de constitución entendemos una ordenación de los objetos en forma de escalera, de modo que los objetos pertenecientes a cada uno de los niveles son constituidos a partir del nivel inferior." (\$ 2)

El procedimiento para constituir un concepto es la 'definición constitucional' por la que todo concepto perteneciente a un nivel es incluido en una clase o en una relación, mediante conceptos provenientes de un nivel anterior ( $\int 7$ ). La teoría de la constitución delimita así el dominio de las proposiciones con sentido, en tanto si pueden formarse proposiciones con sentido acerca de un objeto, entonces este puede ser 'constituido' (\$ 112) —definido mediante conceptos que permiten reducirlo.

El papel de lo a priori en el sistema de constitución toma forma cuando Carnap específica las reglas del lenguaje científico ( $\left(\int 82\right)$. El lenguaje de las teorías científicas se compone de reglas físicas, que conforman el contenido empírico de las teorías, y reglas lógicas de carácter analítico, que conforman el contenido no-empírico y que - a semejanza de los axiomas de coordinación de Reichenbach — son presupuestas en la construcción de las proposiciones con empíricas. Las reglas lógicas funcionan como 'reglas generales de constitución', en cuanto imponen determinaciones para relacionar conceptos en base a comprobaciones empíricas ${ }^{12}$.

Uno de los ejemplos recurrentes de Carnap es la relación de transitividad ( $\int$ 103) El resultado de una comprobación empírica puede arrojar que la relación entre dos conceptos es transitiva o indicar que dos conceptos que tienen una propiedad común están incluidos en la misma clase, pero la relación lógica que se establece entre el resultado empírico y lo que el resultado comprueba - la transitividad o la inclusión en una clase — no vale como empírica, justamente en cuanto es condición previa para la experiencia, y en esa medida es a priori; "pueden ser llamadas reglas a priori en tanto que la constitución y el conocimiento de los objetos se funda en ellas de manera lógica. Pero solamente nos podemos hacer conscientes de dichas reglas con base en la abstracción de una experiencia ya formada, constituida."(\$ 103). Al igual que en Reichenbach, las reglas a priori son constitutivas porque si bien no son empíricas, solamente funcionan si disponen de un material empírico sobre cuál que operar.

${ }^{12}$ Friedman ha señalado con justeza el impacto de esta noción en la filosofía de Kant, "Estas definiciones... son analíticas en sí mismas, y por lo tanto el concepto de conocimiento analítico a priori es suficiente (contra Kant) para proveer una explicación completa de la posibilidad del conocimiento..." $(2007,92)$ 
Para Carnap las reglas de constitución no son proposiciones a priori sino solo determinantes a priori (\$103), y ello en dos sentidos. En primer lugar, no sabemos a priori el contenido de una regla, sino solo su determinación formal — no sabemos si hay relaciones de transitividad, solo sabemos cómo deberían ser en caso de que ocurrieran-, por lo que las reglas por sí solas no proporcionan contenidos de conocimiento, y por ello son a priori pero no sintéticas. En segundo término, la elección de las relaciones básicas de un sistema es 'opcional' en la misma medida en que lo es la elección de los conceptos básicos, por lo que dado un sistema construido sobre determinados conceptos, siempre será posible un sistema alternativo tanto de relaciones como de conceptos ( $\$ 153)$. Para Carnap, si los principios $a$ priori no son necesarios entonces son convencionales; si no son invariantes a la Kant, entonces son resultado de estipulaciones; y esta disyunción fue lo suficientemente fuerte para inhibir la exploración de cualquier otra alternativa ${ }^{13}$.

Las reglas serían conocimiento si supiéramos a priori que nuestra experiencia se rige por lo que una regla indica. Pero hay más de un conjunto de reglas posibles, y no hay ninguna regla que esté necesariamente incrustada en la experiencia, y que por tanto pueda conocerse a priori. El status convencional de las reglas permite a Carnap enfocar su ataque a Kant en términos del convencionalismo de Poincaré: "el conocimiento no tiene más componentes sino estos dos: el convencional y el empírico; es decir, que no hay conocimiento sintético a priori." (\$ 179)

Esto marca una distancia con Reichenbach, quien había considerado a los axiomas de constitución como sintéticos a priori, lo que — de forma muy similar a Poincaré- lo dejaba en posición de cuestionar el status y la función que Kant había conferido a estos juicios, pero no su existencia misma. Reichenbach coincide con Carnap en el carácter formal de los principios a priori, pero sin extraer de ello la negación de su status sintético. Carnap lleva el asunto más lejos, al negar que haya juicios sintéticos a priori, lo que conduce a una concepción formalista y convencionalista de la aprioridad.

La adopción de un a priori convencional en Carnap responde a la misma premisa que en Poincaré, a saber que los principios a priori son producto de estipulaciones respecto a cómo confeccionar un sistema de conocimiento, más que de constricciones impuestas a nuestras capacidades cognitivas. El rol que Carnap

13 Para Mormann, "Carnap propuso un a priori que renunciaba a todas las pretensiones de necesidad, y debía ser interpretado como un principio constitucional puramente hipotético. Este a priori 'movible' iba a convertirse en la fuente primaria para la introducción de componentes convencionales en el dominio del conocimiento físico.” $(2007,52)$ 
atribuye a la estipulación de reglas para la constitución de los conceptos ha llevado a Friedman a sostener que "la constitución entera del mundo externo está determinada por los datos sensoriales, en base a un complicado sistema de convenciones o estipulaciones físicas y metodológicas, que no están destinadas a hacer nada más que codificar las reglas actuales que sigue la ciencia al construir su imagen del mundo físico." $(1999,123)$

La fuerte influencia de Poincaré se entrevé en que para Carnap tanto los conceptos básicos como las reglas lógicas son resultado de decisiones, que permiten la convivencia entre distintas alternativas posibles. Así como para Poincaré contamos con geometrías empíricamente equivalentes entre las que debemos elegir, para Carnap debemos elegir entre distintas opciones de conceptos y reglas básicas.

La idea — común a Carnap y Poincaré- de que los principios que cuentan como a priori dentro de un sistema de conocimiento son estipulados, seguramente sea resultado del particular clima intelectual generado por la revolución de las Gne, y por la presunción de que los científicos podían 'elegir' en cada caso la geometría que fuera más funcional a sus propósitos teóricos. Como ha señalado Friedman, ello supone que la estructura conceptual de la física es lo suficientemente maleable para asimilar la geometría que decidimos elegir, "al final, el marco a priori de la física es producto de una elección convencional o pragmática, basada en la simplicidad y manejabilidad general del total de nuestra teoría física..." (2007, 110). Esta amplitud en la elección de los principios básicos de conocimiento está presente en cada paso de la constitución carnapiana, y especialmente en la consideración de las alternativas para fijar los conceptos básicos del sistema de constitución y definir las reglas lógicas que lo rigen.

A finales de los años 50's cuando Putnam comienza a esbozar su propia idea de lo a priori, la situación es muy diferente. Por un lado, el desarrollo de la teoría general de la relatividad puso en cuestión la idea de que el marco geométrico de los sistemas físicos es libremente elegible, en cuanto la relatividad exige considerar la base geométrica del espacio con una variedad riemanniana de curvatura variable. Por otro lado, la crítica de Quine (1951) a la distinción analítico-sintético impide confinar los principios a priori al dominio de las proposiciones analíticas con la comodidad con que podía hacerlo Carnap en Aufbau. Estas variables permiten comprender por qué en Putnam encontraremos una imagen de lo a priori despojada de componentes convencionales, en favor de considerar que los principios a priori se nos imponen como resultado de los límites de nuestras capacidades cognitivas. En lo que sigue revisaremos la propuesta de Putnam a la luz de estos problemas. 


\section{PUtNAM Y EL $A$ PRIORI CONTEXTUAL}

Según señala Coffa (1991), la propuesta de Reichenbach no permite distinguir entre los principios a priori y los enunciados empíricos, ya que la única diferencia entre unos y otros es que los a priori no pueden contrastarse empíricamente de forma aislada; pero más allá de esto quedan equiparados a los enunciados empíricos. La caracterización del a priori de Putnam busca sanear este problema, enfatizando que 1) los principios que cuentan como a priori no son resultado de una estipulación, sino consecuencia de la imposibilidad de concebir su negación, y 2) los principios a priori solo se vuelven revisables cuando surgen principios alternativos.

Putnam introduce su caracterización del a priori en diálogo con Quine, y particularmente con la tesis de que no hay enunciados inmunes a la revisión (Quine 1951). En principio concede a Quine que la historia de la ciencia conduce a abandonar la idea de que existen principios absolutamente a priori (Putnam 1976), pero no abraza la tesis quineana de que todos los enunciados de un sistema de conocimiento pueden ser revisados en las mismas condiciones. Por el contrario, Putnam entiende que si bien no hay verdades absolutamente a priori, a lo largo de la historia hay principios que han sido asumidos como verdaderos más allá de toda duda. Se trata de principios que solo pueden ser revisados a la luz de una teoría rival, en cuanto quienes los sostienen no conciben condiciones en que puedan ser negados.

Este tipo de enunciados tiene para Putnam un status contextualmente a priori, ya que ofician como verdades necesarias dentro de una esquema conceptual: "hay enunciados en la ciencia que solo pueden ser derrocados por una nueva teoría y no por la sola observación. 'Tales enunciados tienen una clase de 'aprioridad' previa a la intervención de la nueva teoría que los desafía o sustituye: son contextualmente a priori." (1976, 95; tb. $1962 ; 1974 b)^{14}$. Como bien ha señalado Tsou (2010), esta imagen del a priori busca dar cuenta del status especial que ciertos principios básicos tienen en la construcción del conocimiento, y que solo se pone de manifiesto durante las revoluciones científicas.

Al igual que Poincaré, Reichenbach y Carnap, Putnam pretende extraer de las Gne alguna enseñanza sobre los principios básicos de la ciencia. Hasta mediados del siglo XIX los axiomas de Euclides fueron tomados como verdades necesarias a priori, y luego perdieron tal status por gracias el surgimiento de modelos que mostraban el carácter no necesario de la geometría euclidiana (Putnam 1968b).

14 En "Lo analítico y lo sintético" (1962) y en "Science as approximation to truth" (1974b) Putnam afirma que la aprioridad de un enunciado siempre es relativa al esquema conceptual que lo contiene, pero sin emplear aún la expresión a priori contextual. 
Pero quienes asumieron como a priori los axiomas de Euclides durante el período previo al surgimiento de las Gne no efectuaron una elección injustificada, ya que dado el estado del conocimiento geométrico no era posible concebir en qué condiciones la geometría de Euclides podría ser falsa.

Las Gne surgieron como consecuencia de algunos problemas que no resultaban manejables en el marco euclidiano - particularmente el problema del $5^{\circ}$ postulado-, y la geometría de Euclides solo comenzó a ser objeto de revisión cuando las alternativas no euclidianas estuvieron a la vista. Para Putnam esto constituye un rasgo fundamental del a priori contextual; la revisión de una teoría aceptada (y de sus principios a priori) solo se produce cuando hay una alternativa disponible, ya que los indicios contrarios a la teoría inicial solo pueden justificar su revisión si son articulados en un nuevo marco conceptual (Putnam 1962) ${ }^{15}$.

Una objeción clásica a esto es la de Bertolet, para quien la noción de aprioridad contextual es resultado de una confusión entre los procesos epistémicos de los sujetos y las propiedades semánticas de los enunciados, "Los sujetos en cuestión estaban justificados en tomar la geometría euclidiana como compuesta de verdades a priori, esta era la actitud racional a tomar. Equivocados, pero justificados. Es por lo menos confundente e inútil tomar este hecho epistémico acerca de estos sujetos y promoverlo como una propiedad de un enunciado.” $(1988,258)$

No es nuestro propósito discutir en detalle esta cuestión, pero cabe señalar por qué la crítica resulta ineficaz. Putnam no está atribuyendo a los enunciados propiedades de los procesos epistémicos, sino que está reduciendo una propiedad semántica de un enunciado - como el ser a priori- a las condiciones epistémicas en que los sujetos lo adoptan. La aprioridad como propiedad semántica está determinada por el contexto epistémico en que un enunciado vale como a priori, y es justamente la dependencia del contexto epistémico lo que convierte al a priori en contextual ${ }^{16}$.

15 En "The Logic of quantum Mechanics" (1968b) Putnam afirma que la revisión de la geometría de Euclides constituye un caso en el que una verdad tenida como necesaria es abandonada por razones de tipo empírico. Su principal objetivo por esta época es defender el carácter 'empírico' de la lógica, no en el sentido de que los principios lógicos estén expuestos a testeo experimental directo, sino en el sentido de que están expuestos a revisión como consecuencia de los resultados obtenidos por las ciencias naturales; lo mismo vale para la influencia de la teoría general de la relatividad en el cuestionamiento a la geometría de Euclides (Putnam 1965, 1968a, 1968b, 1974a). 16 "Desde el punto de vista de Putnam, es un error tomar a los desarrollos posteriores como implicando que los axiomas de la geometría de Euclides eran tan solo convenciones, o conjeturas empíricas comunes expuestas a refutación. Su status solo puede evaluarse en relación a un horizonte conceptual específico." (Ben-Menahem 2015, 466). 
Putnam sigue a Reichenbach en que los principios a priori son variables y al mismo tiempo constitutivos del conocimiento. Pero toma distancia de la idea de que los principios a priori chocan con la experiencia, adoptando en su lugar una opción más restrictiva en la que los principios únicamente entran en conflicto con otros principios rivales - no con la experiencia de modo directo-y solo lo hacen en contextos de cambio teórico. Aquí la experiencia también ocupa un lugar, en cuanto el desarrollo de un esquema conceptual alternativo responde en buena medida a dificultades empíricas del esquema previo. Pero el punto de Putnam es que los principios a priori solo entran en conflicto con la experiencia por la mediación de un conjunto de principios rivales. Si no hay una alternativa en juego, los principios básicos son reiteradamente protegidos de la revisión, por más recalcitrantes que sean las discrepancias con la experiencia.

Buscando clarificar la relación algo opaca que Reichenbach había dejado entre los principios a priori y la experiencia, Putnam postula que cuando los científicos asumen la aprioridad de un principio este queda protegido del control experimental, al punto que los eventuales resultados contrarios no resultan suficientes para justificar su revisión: "Si un físico hace un cálculo y obtiene un resultado empíricamente erróneo, no sospecha que los principios matemáticos que ha usado en sus cálculos podrían estar equivocados, ni tampoco sospecha que la ley ' $f=m a$ ' pueda ser errónea." (1962, 24)

Esto ubica el frente más fuerte de Putnam contra Carnap y Poincaré, en cuanto el cambio en los principios a priori remite a un conflicto entre marcos teóricos que imponen fuertes restricciones cognitivas a los sujetos que los adoptan, quienes literalmente no pueden pensar fuera de las coordenadas fijadas por los principios que asumen como a priori. Esto no deja margen para la estipulación convencional ni la libre elección ${ }^{17}$. Actualmente aceptamos como necesario a priori el enunciado 'todo número natural tiene un sucesor' (Putnam 1987) no porque lo hayamos estipulado, sino porque no podemos concebir un número natural que no sea sucedido por otro. Solo podríamos revisarlo si comenzara a mostrarse ineficaz para resolver algún problema matemático que consideramos relevante, y si logramos desarrollar una teoría alternativa que implique su negación. En este caso dejaría de ser a priori, pero no como resultado de una elección, sino como

17 Ben-Menahem (2015) ha discutido hasta qué punto la crítica de Putnam hace justicia a las tesis convencionalistas. Si bien no nos detendremos en este punto, buscaremos mostrar que la caracterización putnamiana del a priori logra al menos poner de manifiesto las limitaciones del convencionalismo como marco para explicar el cambio científico. 
consecuencia de cambios en el contexto epistémico. Sólo bajo estas condiciones es posible la revisión de los principios fundamentales de un esquema conceptual, y sólo bajo circunstancias históricas de reorganización del conocimiento es posible su exposición a la crítica ${ }^{18}$.

En la reconstrucción histórica podemos afirmar retrospectivamente que un enunciado tuvo un status contextualmente a priori si durante un cierto período de tiempo no estuvo sujeto a crítica, y si para someterlo a crítica fue necesario desarrollar una teoría alternativa. Pero con nuestras creencias actuales no podemos hacer el mismo tipo de reconstrucción, justamente porque no podemos distinguir cuáles de ellas requerirían el desarrollo de una nueva teoría para ser abandonadas. Como ha señalado Ebbs (2015) Putnam debe aceptar que cualquier enunciado que actualmente consideramos como a priori sea revisado o considerado falso en el futuro, a pesar de que actualmente no podemos concebir cómo ello sería posible.

Putnam (2015) concede esto de buen grado, en cuanto entiende que todos los principios de las teorías científicas son contextualmente a priori. En último término, la posición de Putnam a finales de los 70's es que, dada la fuerte dependencia de los principios a priori respecto del contexto epistémico, el punto de partida de los problemas de la aprioridad es la imposibilidad de cuestionar un enunciado dadas las condiciones impuestas por nuestros esquemas conceptuales, "Si un enunciado tiene la propiedad de que no podemos describir actualmente ninguna circunstancia bajo la cual sería racional abandonarlo, eso seguramente será satisfactorio para la mayoría de nuestros propósitos de argumentación filosófica.” (Putnam 1978, 114)

En una mirada general, Putnam puede listarse sin problemas entre los filósofos que han considerado que los principios a priori con a la vez constitutivos y variables. Sin embargo, la oposición al convencionalismo y la insistencia en que los enunciados a priori imponen constricciones cognitivas a los sujetos, ubica al a priori de Putnam en relación estrecha con Kant y en menor medida con Reichenbach, pero en las antípodas de las concepciones que consideran los principios a priori como objetos de una elección. Dedicaremos la conclusión a explorar con algo más de detalle el alcance de esta pervivencia kantiana en el pensamiento de Putnam.

${ }^{18}$ En "There is at Lesat one a priori Truth" (1978), Putnam complejiza algo más su posición, al señalar que hay por lo menos un enunciado que puede considerado como a priori absoluto y no contextual. Este es el denominado principio mínimo de no-contradicción, a saber: no todo enunciado es verdadero y falso. Pero en una nota al final del texto toma distancia de cualquier a priori absoluto, y mantiene dicha posición en escritos posteriores $(1981,2015)$. 


\section{CONCLUSIÓN}

Todos los autores que hemos revisado concuerdan en que los principios a priori no son necesarios, sino que varían con el tiempo. También concuerdan en que son constitutivos del conocimiento, en cuánto fijan las coordenadas cognitivas para el acceso a un dominio de objetos. Pero difieren en la forma en la que se da el proceso de constitución, y especialmente en el status de los principios a priori. Para Poincaré y para Carnap con convenciones libradas a la elección de los sujetos, para Reichenbach no son convencionales en cuanto están expuestos a control empírico, y para Putnam ni son convencionales ni están expuestos a control empírico directo.

Es necesario introducir algunos elementos históricos para comprender estas diferencias. La proliferación de modelos geométricos alternativos durante la revolución de las Gne fertilizó la idea de que tanto los principios de la geometría como elección de la base métrica de la física son asunto de estipulación, base de las lecturas convencionalistas de lo a priori introducidas por Poincaré, Schlick y Carnap entre otros. Pero el desarrollo de la relatividad general y la adopción de una variedad de Riemann como base métrica de la física generó dificultades irreversibles para este programa, por lo que la defensa del carácter no-convencional de lo a priori en el primer Reichenbach y en Putnam constituye una respuesta a los callejones sin salida del convencionalismo.

Ben-Menahen (2015) recuerda que el convencionalismo destaca el rol de la decisión humana, lo que supone que nuestros esquemas de conocimiento (y por supuesto sus principios básicos), son resultado de cómo elegimos organizar nuestras herramientas cognitivas. En la mirada de Putnam (1974b) el convencionalismo cae en una suerte de ilusión de libertad que contempla que el estatus a priori de ciertos principios es resultado de la dinámica evolutiva del conocimiento y no de las decisiones de los sujetos. Esto permite a Putnam rescatar el carácter no psicológico del a priori, en cuanto la revisión de principios básicos no depende de las intenciones de un sujeto que elige, sino de las constricciones impuestas por los sistemas de conocimiento, "no es una mera cuestión de lo que alguna gente puede imaginar o no imaginar; es cuestión de lo que, dado un esquema conceptual, uno sabe cómo falsar o al menos disconfirmar. Antes de Lobachevski, Riemann y otros, nadie sabía cómo disconfirmar la geometría de Euclides, ni siquiera sabían si algo podría disconfirmarla." (Putnam 1994, 251; tb. 2015)

Tanto Reichenbach como Putnam niegan el convencionalismo, y ofrecen una propuesta que reactiva la idea kantiana de los principios a priori como límite del pensamiento posible. En el caso de Reichenbach esta actitud no pasa de un 
romance de verano, ya que poco después abraza el convencionalismo; pero en Putnam dará lugar a una imagen sumamente articulada de lo a priori como condición de posibilidad del conocimiento, y como un límite cognitivo que solo puede rebasarse en contextos de cambio teórico. No parece haber mejor opción que denominar 'trascendental' a esta forma marcadamente kantiana de comprender los principios a priori.

Tal como ha señalado Carus $(2007,117)$, Kant pensaba que en cuestiones de geometría no tenemos elección, ya que la idealidad del tiempo y el espacio marca el límite de las condiciones en que podemos pensar y conocer. Putnam mantiene esta impronta al señalar que solo podemos elegir los principios a priori en contextos específicos de revoluciones científicas, lo que produce una narrativa diacrónica del a priori que solo asoma en Reichenbach y está ausente en los abordajes convencionalistas. De este modo, la concepción de Putnam se estructura a partir de la idea kantiana de que los principios a priori constituyen los objetos, en el sentido fuerte de que solo a través de dichos principios los objetos pueden ser pensados. Pero la constitución no es resultado de que decidimos trabajar con determinados principios, ya que los principios fijan las condiciones de posibilidad del conocimiento de un modo tal que no es posible optar por condiciones alternativas.

El a priori putnamiano mantiene naturalmente el carácter constitutivo, pero también rehabilita el status trascendental que Kant había conferido a los principios a priori, si entendemos por trascendentales los principios que fijan las condiciones de posibilidad del conocimiento. En cuanto imponen constricciones cognitivas de las que los sujetos no pueden escapar por un acto deliberado, los a priori de Putnam son tan trascendentales como los de Kant. La diferencia naturalmente estriba en que el a priori kantiano fija condiciones de conocimiento invariables y atemproales, mientras que el a priori putnamiano fija condiciones variables y contextuales. Pero desde el momento en que en ambos casos los principios a priori no son revocables por los sujetos — salvo en contexto de revolución científica-, se trata de principios que cumplen una función trascendental.

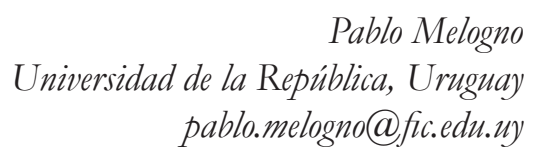




\section{BIBLIOGRAFÍA}

Ben-Menahen, Y. (2015): "Revisiting the Refutation of Conventionalism", in L. Hahn \& R. Auxier (eds.), The Philosophy of Hilary Putnam, Illinois: Open Court, pp. 451-478. Bertolet, R. (1988): "Putnam on the a priori", Philosophia, vol. 18, no 2-3, pp. 253-263. CARNAP, R. (1928): La construcción lógica del mundo, México: UNAM. [1988]

Carus, A. (2007): Carnap and the Twentieth Century Thought, Cambridge-UK: Cambridge University Press.

Coffa, J. A. (1991): The semantic tradition from Kant to Carnap, Cambridge-Mass: Cambridge University Press.

DiSalle, R. (2006): Understanding space-time, New York: Cambridge University Press.

EBBS, G. (2015): "Putnam and the Contextually A Priori", in L. Hahn \& R. Auxier (eds.), The Philosophy of Hilary Putnam, Illinois: Open Court, 2015, pp. 389-411.

FolinA, J. (2014): "Poincaré and the Invention of Convention", in M. de Paz \& R. DiSalle (eds.), Poincaré, Philosopher of Science: Problems and Perspectives, New York: Springer, 25-45.

Friedman, M. (1999): Reconsidering Logical Positivism, Cambridge-UK: Cambridge University Press.

Friedman, M. (2002): "Kant, Kuhn, and the Rationality of Science", Philosophy of Science, vol. $69, \mathrm{n}^{\circ} 2$, pp. 171-190.

FrIEDMAN, M. (2007): "Coordination, Constitution and Convention: the Evolution of the a priori in Logical Empiricism”, in A. Richardson \& T. Uebel (eds.), The Cambridge Companion to Logical Positivism, New York, Cambridge University Press, pp. 91-116.

KANT, I. (1781): Crítica de la Razón Pura, Madrid: Alfaguara. [2004]

Mormann, T. (2007): “Geometrical leimotifs in Carnap's early Philosophy", in M. Friedman \& E. Creath (eds.), The Cambridge Companion to Carnap, Cambridge-UK: Cambridge University Press, 2007, pp. 43-64.

PATY, M. (1992): "Physical Geometry and Special Relativity: Einstein and Poincaré", in L. Boi, D. Flament \& J. M. Salanski (eds.), 1830-1930: un siècle de géométrie, de C. F. Gauss et B. Riemann à H. Poincaré et E. Cartan. Epistémologie, histoire et mathématiques, Berlin: Springer-Verlag, pp.126-149.

Peláez, A. (2008a): Carnap, México: Universidad Autónoma Metropolitana.

PELÁEZ, A. (2008b): El a priori constitutivo: historia y prospectiva, México: Universidad Autónoma Metropolitana/ Anthropos.

Poincaré, H. (1905): Science and Hypothesis, London: The Walter Scott Publishing Co.

Putnam, H. (1962): Lo analítico y lo sintético, México: UNAM. [1983]

Putnam, H. (1965): "A Philosopher Looks at Quantum Mechanics", in Mathematics, Matter and Method, Philosophical Papers, vol. 1, Cambridge-Mass: Cambridge University Press, pp. 130-158. [1975]

Putnam, H. (1968a): "Is logic empirical?”, in R. Cohen \& M. Wartofsky (eds.), Boston Studies in the Philosophy of Science, vol. 5, Dordrecht: D. Reidel, pp. 216-241. 
PutNam, H. (1968b): “The Logic of Quantum Mechanics", in Mathematics, Matter and Method, Philosophical Papers, vol. 1, Cambridge-Mass: Cambridge University Press, pp. 174-197. [1975]

PutNAm, H. (1974a): "How to think quantum-logically", Synthese vol. 29, no 1-4, pp. 55-61. PutNAM, H. (1974b): "Introduction: Science as approximation to truth", in Mathematics, Matter and Method, Philosophical Papers, vol. 1, Cambridge-Mass.: Cambridge University Press, pp. VII-XIV. [1975]

Putnam, H. (1974c): “The Refutation of Conventionalism”, Noûs vol. 8, n¹, pp. 25-40.

Putnam, H. (1976): “Two dogmas revisited”, in Realism and Reason, Philosophical Papers, vol. 3, Cambridge-Mass.: Cambridge University Press, pp. 87-97. [1983]

PutNam, H. (1978): “There is at least one a priori truth", in Realism and Reason, Philosophical Papers, vol. 3, Cambridge-Mass.: Cambridge University Press, pp. 98-114. [1983]

PutNam, H. (1979): "Analyticity and Apriority: Beyond Wittgenstein and Quine", in Realism and Reason, Philosophical Papers, vol. 3, Cambridge-Mass.: Cambridge University Press, pp. 115-138. [1983]

PutNam, H. (1981): Razón, verdad e historia, Madrid: Tecnos. [1988]

Putnam, H. (1994): "Rethinking Mathematical Necessity", in Words and life. CambridgeMass.: Harvard University Press, pp. 245-263.

Putnam, H. (2015): "Reply to Gary Ebbs", in L. Hahn \& R. Auxier (eds.), The Philosophy of Hilary Putnam, Illinois: Open Court, 2015, pp. 412-417.

QuINE, W.O. (1951): "Dos dogmas del empirismo", en Desde un punto de vista lógico. Buenos Aires: Orbis, pp. 49-81. [1984]

ReICHenBaCH, H. (1920): The Theory of Relativity and a Priori Knowledge, Berkeley: University of California Press. [1965]

RyCKMAN, T. (1996): "Einstein Agonists: Weyl and Reichenbach on Geometry and the General Theory of Relativity", in R. Giere \& A. Richardson (eds.), Origins of Logical Empiricism, Minneapolis: University of Minnesota Press, pp. 165-209.

Ryckman, T. (2003): “Two Roads from Kant: Cassirer, Reichenbach and General Relativity", in P. Parrini, W. Salmon \& M. Salmon (eds.), Logical Empiricism. Historical and Contemporary Perspectives, Pittsburgh: University of Pittsburgh Press, pp. 159-193.

SCHlick, M. (1922): Space and Time in Contemporary Physics, in Moritz. Schlick: Philosophical Papers, vol. 1, Dordrecht, Reidel, pp. 207-269.

Tsou, J. Y. (2010): "Putnam's account of apriority and scientific change: its historical and contemporary interest”, Synthese, vol. 176, n 3, pp. 429-445. 